International Mathematical Forum, 2, 2007, no. 27, 1305 - 1310

\title{
An Application of the Fibonacci Length
}

\author{
H. Doostie \\ Mathematics Department, Teacher Training University \\ 49 Mofateh Ave., Tehran 15614, Iran \\ doostih@saba.tmu.ac.ir
}

M. Hashemi

Mathematics Department, Faculty of Science

Guilan University, P.O. Box 451, Rasht, Iran

$m_{-}$hashemi@guilan.ac.ir

\begin{abstract}
For every integer $n \geq 2$ we define the Fibonacci class of degree 2 of 2-generated groups, and study certain properties of this class. Our method of study is applicable for the groups with more than two generators. This investigation is indeed an application of $F L_{A}(G)$, the well-known Fibonacci length of the finite group $G=\langle A\rangle$, where $A=\left\{a_{1}, a_{2}, \ldots, a_{k}\right\}$.
\end{abstract}

Mathematics Subject Classification: 20F05

Keywords: Fibonacci length, finitely presented groups

\section{Introduction}

Since 1990 the Fibonacci length has been studied and calculated for certain classes of finite groups (one may see $[1,2,3,4,5,6]$ for examples), where the least positive integer $l$ is called the Fibonacci length of the group $G=\langle A\rangle$ with respect to the generating set $A=\left\{a_{1}, a_{2}, \ldots, a_{n}\right\}$, denoted by $L E N_{A}(G)$, if it is the period of the sequence

$$
x_{1}=a_{1}, x_{2}=a_{2}, \ldots, x_{n}=a_{n}, \quad x_{i}=x_{i-n} . x_{i-n+1} \ldots x_{i-2} . x_{i-1}, \quad i \geq n+1,
$$

of the elements of $G$. When it is clear which generating set of the group $G$ is being considered we will write $L E N(G)$ for $L E N_{A}(G)$. Our notation are standard. The Fibonacci sequence of numbers, $\left\{F_{n}\right\}_{-\infty}^{\infty}$, defined by 


$$
\left\{\begin{array}{l}
F_{n}=F_{n-2}+F_{n-1}, \quad n \geq 0 \\
F_{n}=F_{n+2}-F_{n+1}, \quad \text { otherwise }
\end{array}\right.
$$

where we seed the sequence with $F_{0}=0$ and $F_{1}=1$. Following Wall ([8]), we use the notation $k(n)$ to denote the fundamental period of this sequence. We will also use $C(G)$ and $T(G)$ for the complexity and T-invariant of a finitely presented group $G$, respectively.

In this paper we give an application of the Fibonacci length on the classification of groups, by using $C(G)$ and $T(G)$. This application is the first theoretical result of this notion after all of its nice numerical results. Following Pervova ([7]) recall two definitions:

Definition 1.1 Let $\left\langle a_{1}, a_{2}, \ldots, a_{n} \mid r_{1}, r_{2}, \ldots, r_{m}\right\rangle$ be a presentation of a group $G$. The length of this presentation is defined to be $\left|r_{1}\right|+\ldots\left|r_{m}\right|$ where, $\left|r_{i}\right|$ is the length of the word $r_{i}$ in the alphabet $a_{1}^{ \pm 1}, a_{2}^{ \pm 1}, \ldots, a_{n}^{ \pm 1}$. The Complexity is often called the minimum length (of a group). Also $\left|r_{i}\left(a_{j}\right)\right|$ is defined to be the sum of the powers of the generator $a_{j}$ in the relator $r_{i}$, for every $i$ and $j$.

Definition 1.2 The T-invariant of a finitely presented group $G, T(G)$ is the minimum number $t$ such that $G$ admits a presentation with $t$ relations of length 3 and an arbitrary number of relations of length at most 2.

By the above considerations we now give the following definitions.

Definition 1.3 For a 2-generated non-abelian group $G$ let

$$
K_{*}(G)=\min \left\{L E N_{A}(G)|G=\langle A\rangle, \quad| A \mid=2, L E N_{A}(G)<\infty\right\} .
$$

Then for every integer $n \geq 2$, the class of all 2-generated groups $G$ satisfying $K_{*}(G) \mid n$ will be denoted by $F L_{n}$, is called the Fibonacci class of length $n$.

In what follows we investigate the class $F L_{n}$ for 2-generated groups and our method of investigation will be applicable for the non-abelian groups with more than two generators.

Definition 1.4 For a 2-generated group $G=\langle A\rangle$, where $A=\{a, b\}$, the Co-Fibonacci orbit of $G$ with respect to $A$, is the sequence

$$
y_{1}=b, \quad y_{2}=a, \quad y_{i+1}=y_{i-1} y_{i}^{-1}, \quad i \geq 2 .
$$

By this definition the period of the sequence $\left\{x_{i}\right\}$ is equal to the period of the sequence $\left\{y_{i}\right\}$. Obviously, if $G \in F L_{n}$ then $y_{i}=x_{n+3-i}$, for every $i \geq 1$. 


\section{A classification method}

Firstly we consider the following preliminary lemma.

Lemma 2.1 By the above notations $\left|x_{k}\right|=F_{k},\left|y_{k}\right|=F_{k},\left|x_{k}(a)\right|=F_{k-2}$, $\left|x_{k}(b)\right|=F_{k-1},\left|y_{k}(a)\right|=(-1)^{k} F_{k-1}$ and $\left|y_{k}(b)\right|=(-1)^{k-1} F_{k-2}$.

Proof. To prove $\left|x_{k}\right|=F_{k}$ we use an induction method. Indeed, $\left|x_{1}\right|=F_{1}$ and $\left|x_{2}\right|=F_{2}$ are true and then $x_{k}=x_{k-2} x_{k-1}$ gives that $\left|x_{k}\right|=\left|x_{k-2}\right|+\mid$ $x_{k-1} \mid=F_{k}$. Other statements may be proved in a similar way.

The following proposition is of interest to consider and one may see the proof in Pervova ([7]).

Proposition 2.2 For a finitely presented group $G$,

(i) $T(G)=\min \left\{\sum_{i=1}^{m} \max \left\{\left|r_{i}\right|-2,0\right\} \mid G=\left\langle a_{1}, a_{2}, \ldots, a_{n} \mid r_{1}, r_{2}, \ldots, r_{m}\right\rangle\right\}$.

(ii) $C(G) \geq \log _{2}^{\left|\operatorname{Tor}\left(G / G^{\prime}\right)\right|}$, where Tor $(H)$ is used for the torsion part of an abelian group $H$.

Proposition 2.3 For a finitely presented group $G=\langle X \mid R\rangle, G \in F L_{n}$ if and only if $G=\langle a, b \mid R\rangle$ where

$$
\left\{x_{[n / 2]+1} \cdot y_{[(n+1) / 2]+2}^{-1}, x_{[n / 2]+2} \cdot y_{[(n+1) / 2]+1}^{-1}\right\} \subseteq R .
$$

Proof. Let $G \in F L_{n}$. Then there exists a generating set $A=\langle a, b\rangle$ for $G$ such that $K_{*}(G)=\operatorname{LEN}_{A}(G)$ and $K_{*}(G) \mid n$. By using the relations $x_{n+1}=x_{n-1} x_{n}$ and $y_{n+1}=y_{n-1} y_{n}^{-1}$ we get

$$
\left\{\begin{array}{l}
x_{n+1}=a=y_{2} \\
x_{n+2}=b=y_{1}
\end{array}\right.
$$

and then we get $x_{n}=b a^{-1}=y_{3}$. Continuing this process yields

$$
\left\{x_{[n / 2]+1} \cdot y_{[(n+1) / 2]+2}^{-1}, x_{[n / 2]+2} \cdot y_{[(n+1) / 2]+1}^{-1}\right\} \subseteq R .
$$

Conversely, let $G=\langle X \mid R\rangle$ and the above relation holds, then we get

$$
\left\{\begin{array}{l}
x_{[n / 2]+1}=y_{[(n+1) / 2]+2} \\
x_{[n / 2]+2}=y_{[(n+1) / 2]+1}
\end{array}\right.
$$

or,

$$
\left\{\begin{array}{l}
x_{[n / 2]+1} \cdot x_{[n / 2]+2}=y_{[(n+1) / 2]+2} \cdot y_{[n / 2]+1} \\
x_{[n / 2]+2}=y_{[(n+1) / 2]+1} .
\end{array}\right.
$$

Consequently, $x_{[n / 2]+3}=y_{[(n+1) / 2]}$ and $x_{[n / 2]+2}=y_{[(n+1) / 2]+1}$. Finally, this process yields $x_{n+2}=y_{1}=b$ and $x_{n+1}=y_{2}=a$. So, $G \in F L_{n}$.

As a result of this proposition and using the well-known von-Dyke's theorem (on the presentation of groups) we get: 
Corollary 2.4 For a finitely presented group $G, G \in F L_{n}$ if and only if $G$ is a homomorphic image of the group $\left\langle a, b \mid r_{1}, r_{2}\right\rangle$ where,

$$
r_{1}=x_{[n / 2]+1} \cdot y_{[(n+1) / 2]+2}^{-1}, r_{2}=x_{[n / 2]+2} \cdot y_{[(n+1) / 2]+1}^{-1} .
$$
Then,

Proposition 2.5 Let $G=\left\langle a, b \mid r_{1}=x_{[n / 2]+1} \cdot y_{[(n+1) / 2]+2}^{-1}, r_{2}=x_{[n / 2]+2} \cdot y_{[(n+1) / 2]+1}^{-1}\right\rangle$.

(i) for even values of $n, T(G) \leq 2 F_{(n / 2)+3}-4$,

(ii) for odd values of $n, T(G) \leq F_{(n+9) / 2}-4$.

Proof. For even values of $n$ we get $\left|r_{1}\right|=\left|r_{2}\right|=F_{(n / 2)+1}+F(n / 2)+3=$ $F_{(n / 2)+3}$. Then (i) follows by using the Proposition 2. A similar method for odd values of $n$ shows that $\left|r_{1}\right|=F_{(n+1) / 2}+F_{(n+5) / 2}$ and $\left|r_{2}\right|=2 F_{(n+3) / 2}$. Therefore, $T(G) \leq F_{(n+5) / 2}+F_{(n+7) / 2}-4=F_{(n+9) / 2}-4$.

\section{Corollary 2.6}

(i) A finitely presented group $G$ belongs to the class $F L_{3}$ if and only if $G=\langle a, b \mid R\rangle$ where, $\left\{a b a b^{-1}, b a b a^{-1}\right\} \subseteq R$. In particular, $Q_{8} \in F L_{3}$.

(ii) A finitely presented group $G$ belongs to the class $F L_{6}$ if and only if $G=$ $\langle a, b \mid R\rangle$ where, $\left\{a^{2} b a^{2} b^{-1}, b^{2} a b^{2} a^{-1}\right\} \subseteq R$. In particular, $D_{2 n}, D_{\infty} \in F L_{3}$.

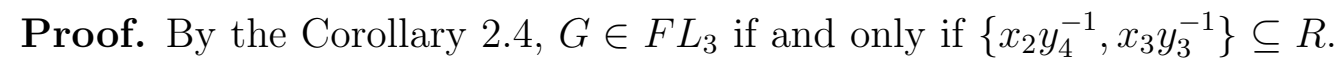
And obviously this is equivalent to $\left\{b\left(a^{2} b^{-1}\right)^{-1}, a b\left(b a^{-1}\right)^{-1}\right\} \subseteq R$. Now, (i) follows by a simple manipulation of the relations. The assertion (ii) may be proved in a similar way and $G \in F L_{6}$ is equivalent to $\left\{x_{4} y_{5}^{-1}, x_{5} y_{4}^{-1}\right\} \subseteq R$, i.e.; $\left\{b a b\left(b a^{-1} b a^{-2}\right)^{-1}, a b^{2} a b\left(a^{2} b^{-1}\right)^{-1}\right\} \subseteq R$. The result is now immediate.

For the particular cases consider the presentations $Q_{8}=\left\langle a, b \mid a^{4}, a^{2} b^{-2}, b^{-1} a b a\right\rangle$, $D_{2 n}=\left\langle a, b \mid a^{2}, b^{2},(a b)^{n}\right\rangle$ and $D_{\infty}=\left\langle a, b \mid a^{2}, b^{2}\right\rangle$.

\section{Conclusion}

The relation matrix of the quotient groups $G / G^{\prime}$, for every groups $G \in F L_{n}$ depends on the Fibonacci numbers. Indeed, we have:

Proposition 3.1 Let $G=\left\langle a, b \mid x_{[n / 2]+1}=y_{[(n+1) / 2]+2}, x_{[n / 2]+2}=y_{[(n+1) / 2]+1}\right\rangle$. 
Then $M\left(G / G^{\prime}\right)$, the relation matrix of the group $G / G^{\prime}$ is equal to:

$$
\begin{aligned}
& M_{1}=\left(\begin{array}{ll}
F_{n / 2} & 0 \\
0 & 5 F_{n / 2}
\end{array}\right), \quad \text { if } n \equiv 0 \bmod 4, \\
& M_{2}=\left(\begin{array}{ll}
F_{n / 2-1}+F_{n / 2+1} & 0 \\
0 & F_{n / 2-1}+F_{n / 2+1}
\end{array}\right), \quad \text { if } n \equiv 2 \bmod 4 \text {, } \\
& M_{3}=\left(\begin{array}{ll}
F_{(n-3) / 2}+F_{(n+3) / 2} & -F_{(n-3) / 2} \\
-F_{(n-3) / 2} & -F_{(n+3) / 2}
\end{array}\right), \quad \text { if } n \equiv 1 \bmod 4 \text {, } \\
& M_{4}=\left(\begin{array}{ll}
-2 F_{(n-1) / 2} & F_{(n+3) / 2} \\
F_{(n+3) / 2} & -F_{(n-3) / 2}
\end{array}\right), \quad \text { if } n \equiv 3 \bmod 4 .
\end{aligned}
$$

Proof. We consider two cases for $n$ and use the Lemma 2.1. If $n$ is even then

$$
\begin{array}{ll}
\left|r_{1}(a)\right|=F_{n / 2-1}+(-1)^{n / 2+1} F_{n / 2+1}, & \left|r_{1}(b)\right|=F_{n / 2}+(-1)^{n / 2} F_{n / 2}, \\
r_{2}(a) \mid=F_{n / 2}+(-1)^{n / 2} F_{n / 2}, & \left|r_{2}(b)\right|=F_{n / 2+1}+(-1)^{n / 2+1} F_{n / 2-1} .
\end{array}
$$

So the result follows in two cases when $n \equiv 0$ or $2 \bmod 4$.

For the odd values of $n$ we get

$$
\begin{array}{ll}
\left|r_{1}(a)\right|=F_{(n-3) / 2}+(-1)^{(n-1) / 2} F_{(n+3) / 2}, & \left|r_{1}(b)\right|=F_{(n-1) / 2}+(-1)^{(n+1) / 2} F_{(n+1) / 2}, \\
\left|r_{2}(a)\right|=F_{(n-1) / 2}+(-1)^{(n-3) / 2} F_{(n+1) / 2}, & \left|r_{2}(b)\right|=F_{(n+1) / 2}+(-1)^{(n-1) / 2} F_{(n-1) / 2} .
\end{array}
$$

If $n \equiv 3 \bmod 4$, then

$$
M\left(G / G^{\prime}\right)=\left(\begin{array}{cc}
F_{(n-3) / 2}-F_{(n+3) / 2} & F_{(n-1) / 2}+F_{(n+1) / 2} \\
F_{(n-1) / 2}+F_{(n+1) / 2} & F_{(n+1) / 2}-F_{(n-1) / 2}
\end{array}\right)
$$

which is equivalent to the matrix $M_{4}$. And if $n \equiv 1 \bmod 4$, then we get

$$
M\left(G / G^{\prime}\right)=\left(\begin{array}{cc}
F_{(n-3) / 2}+F_{(n+3) / 2} & F_{(n-1) / 2}-F_{(n+1) / 2} \\
F_{(n-1) / 2}-F_{(n+1) / 2} & F_{(n-1) / 2}+F_{(n+1) / 2}
\end{array}\right)
$$

which is equivalent to the matrix $M_{3}$. This completes the proof.

As an example we may consider the group $H=\left\langle a, b \mid a^{2} b a^{2}=b, b^{2} a b^{2}=a\right\rangle$ and by the propositions 2.2 and 3.1 we get $\left|H / H^{\prime}\right|=\left(F_{2}+F_{4}\right)^{2}=16$ and $C(H) \geq 4$.

Corollary 3.2 For every even values of $n$ if $G \in F L_{n}$ then $\left|G / G^{\prime}\right|$ divides $5 F_{n / 2}^{2}$ or $\left(F_{n / 2-1}+F_{n / 2+1}\right)^{2}$ if $n \equiv 0 \bmod 4$ or $n \equiv 2 \bmod 4$, respectively.

Proof. By the Corollary 2.4 and the Proposition 3.1. 


\section{References}

[1 ] H. Aydin and G. C. Smith, Finite p-quotients of cyclically presented groups, J. London Math. Soc., 49 (1994), 83-92.

[2 ] C. M. Campbell, H. Doostie and E. F. Robertson, Fibonacci length of generating pairs in groups, In Applications of the Fibonacci Numbers, 3, 27-35. Ed, G. A. Bergum, A. N. Philippou, and A. F. Horadam, Dordrecht: Kluwer, 1990.

[3 ] C. M. Campbell, P. P. Campbell, H. Doostie and E. F. Robertson, Fibonacci length for certain metacyclic groups, Algebra Colloquium, 11:2 (2004), 215-222.

[4 ] C. M. Campbell, P. P. Campbell, H. Doostie and E. F. Robertson, On the Fibinacci length of powers of dihedral groups, In Applications of the Fibonacci Numbers, 9, 69-85, Ed. G. A. Bergum and A. F. Horadam, Dordrecht: Kluwer, 2004.

[5 ] H. Doostie and C. M. Campbell, Fibonacci length of automorphism groups involving Tribonacci numbers, Vietnam J. of Math., 28:1 (2000), 57-65.

[6 ] H. Doostie and R. Golamie, Computing on the Fibonacci length of finite groups, Internat. J. Appl. Math., 4:2 (2000), 149-156.

[7 ] E. Pervova and C. Petronio, Complexity and T-invariant of abelian and Milnor groups, arXiv: math. GT/0412187 V19 December 2004.

[8 ] D. D. Wall, Fibonacci series modulo m, Amer. Math. Monthly, 67 (1960), 525-532.

Received: September 16, 2006 